\title{
Case - Prostate-specific antigen bounce: A pitfall in prostate-specific membrane antigen positron emission tomography/computed tomography interpretation
}

Victor Polins Pedro ${ }^{1}$; Golmehr Sistani ${ }^{2,3}$; Irina Rachinsky ${ }^{3}$; Katherine Zukotynski ${ }^{2,4}$; Glenn Bauman $^{5}$; Wei Liu ${ }^{5}$

${ }^{1}$ Schulich School of Medicine and Dentistry, London, ON, Canada; ${ }^{2}$ Department of Radiology, Department of Imaging, London Health Sciences Centre and Western University, London, ON, Canada; ${ }^{3}$ Department of Nuclear Medicine, Department of Imaging, London Health Sciences Centre and Western University, London, ON, Canada; ${ }^{4}$ Departments of Medicine and Radiology, McMaster University, Hamilton, ON, Canada; ${ }^{5}$ Department of Oncology, Division of Radiation Oncology, London Health Sciences Centre and Western University, London, ON, Canada

Cite as: Pedro VP. Sistani G, Rachinsky I, et al. Case - Prostate-specific antigen bounce: A pitfall in prostate-specific membrane antigen positron emission tomography/computed tomography interpretation. Can Urol Assoc J 2021 May 11; Epub ahead of print. http://dx.doi.org/10.5489/cuaj.7257

Published online May 11, 2021

Correspondence: Dr. Golmehr Sistani, Department of Radiology, Department of Imaging, London Health Sciences Centre and Western University, London, ON, Canada; golmehr.sistani@1hsc.on.ca

$* * *$

\section{Introduction}

Prostate-specific antigen (PSA) "bounce," a transient rise in PSA post-therapy, can happen after either brachytherapy (BT) or external beam radiation therapy (EBRT); however, it is more common in the former case $(1,2)$. In general, PSA bounce tends to occur within 2-3 years of treatment, rarely exceeds levels of 2-3ng/ml above the nadir PSA and tends to resolve over 6-12 months (2). Studies have shown that PSMA PET/CT (Prostate-specific membrane antigen positron emission tomography/Computational Tomography) has a high sensitivity and specificity for the detection of prostate cancer recurrence and can potentially guide therapy (3). As salvage treatment (such as local or systemic salvage) may have associated morbidity, it is important to exclude the possibility of PSA bounce before proceeding with salvage treatment. We describe a case of clinically suspected local failure with initial PSA rise and suspicious PSMA PET/CT finding post therapy that was actually related to a PSA bounce, emphasizing the need for caution in the evaluation of men where a PSA bounce may be a confounding phenomenon. 


\section{Case report}

A 51-year-old male with intermediate risk prostate cancer, clinical T2a, Gleason score $7(3+4)$ had low dose rate (LDR) Iodine-125 BT. His PSA at the time of diagnosis was $7.5 \mathrm{ng} / \mathrm{mL}$ falling to 4.8 and then $2.45 \mathrm{ng} / \mathrm{mL}$ at 3- and 9-months, respectively, post-BT. However, his PSA rose to $2.91 \mathrm{ng} / \mathrm{mL}, 15$ months after therapy and continued to rise on follow-up to $5.8 \mathrm{ng} / \mathrm{mL}$ approximately 30 months post BT. Disease recurrence was suspected. A CT scan of abdomen and pelvis, multi-parametric Magnetic Resonance Imaging (mpMRI) of the pelvis and bone scan were all negative. PSMA PET/CT showed mild focal uptake in the right prostate apex equivocal for recurrence (Figure 1). In the context of rising PSA, this was clinically considered concerning for recurrence. Upon urology consultation, the decision was made to proceed with biopsy and then potentially high-intensity focused ultrasound (HIFU). However, a repeat PSA, before the biopsy and HIFU therapy or any systemic therapy, showed a spontaneous reduction in PSA to $3.23 \mathrm{ng} / \mathrm{mL}$. On further follow-up, the PSA continued to decrease, becoming undetectable 65 months post-therapy in keeping with a benign PSA bounce post BT (Figure 2).

\section{Discussion}

In general, an increase $\geq 0.2 \mathrm{ng} / \mathrm{ml}$ above the nadir within 2-3 months of therapy with subsequent decline has been widely accepted as a PSA bounce (4). A recent meta-analysis of 26,258 patients post-radiation (BT or EBRT) showed PSA bounce in one-third of cases with pooled mean amplitude of $1.3 \mathrm{ng} / \mathrm{mL}$ and time to occurrence of 18 months (5). Usually, serial PSA levels for 1-2 years after the initial rise can differentiate biochemical recurrence from PSA bounce.

In this case, the increase in PSA continued over a longer period of time and reached a higher level than one might typically expect with a benign PSA bounce after BT. PSMA PET/CT also has limitations in the detection of local recurrence since benign prostatic pathologies such as focal prostatitis, benign prostatic hyperplasia and even normal prostate can show areas of increased uptake $(6,7)$. Some strategies such as interval increased activity on delayed PSMA PET/CT scan can suggest malignancy in equivocal cases (8). Also, studies have shown that imaging and biopsy results can evolve post-radiation $(9,10)$. While PSMA PET/CT is well studied for the detection of extra-prostatic recurrence with known sources of false positives, there is little information regarding serial changes in PSMA-avid disease on PET/CT after primary radiation and the relationship of these changes to intra-prostatic control are unknown $(11,12)$. Until larger studies become available, we suggest caution in interpreting isolated local (prostate gland) recurrence detected on PSMA PET/CT at early time points (less than 2-3 years) post radiotherapy. At early time points post radiotherapy, continued serial PSA surveillance with deferred local salvage is warranted. If local salvage is contemplated, a confirmatory biopsy with clear confirmation of local recurrence prior to therapy is recommended. 


\section{References}

1. Caloglu M, Ciezki J. Prostate-specific antigen bounce after prostate brachytherapy: review of a confusing phenomenon. Urology 2009; 74:1183-90.

2. Kubo K, Wadasaki K, Kimura T, et al. Clinical features of prostate-specific antigen bounce after 125I brachytherapy for prostate cancer. J Radiat Res 2018; 59:649-655.

3. Trabulsi EJ, Rumble RB, Jadvar H, et al. Optimum Imaging Strategies for Advanced Prostate Cancer: ASCO Guideline. J Clin Oncol 2020; 38:1963-1996.

4. Caloglu M, Ciezki JP, Reddy CA, et al. PSA bounce and biochemical failure after brachytherapy for prostate cancer: a study of 820 patients with a minimum of 3 years of follow-up. Int J Radiat Oncol Biol Phys 2011; 80:735-41.

5. Darwis NDM, Oike T, Kubo N, et al. Characteristics of PSA Bounce after Radiotherapy for Prostate Cancer: A Meta-Analysis. Cancers (Basel) 2020; 12:2180.

6. Shaygan B, Zukotynski K, Bénard F, et al. Canadian Urological Association best practice report: Prostate-specific membrane antigen positron emission tomography/computed tomography (PSMA PET/CT) and PET/magnetic resonance (MR) in prostate cancer. Can Urol Assoc J 2021;15.

7. Shetty D, Patel D, Le K, et al. Pitfalls in Gallium-68 PSMA PET/CT Interpretation-A Pictorial Review. Tomography 2018; 4:182-193.

8. Sistani G, Metser U, Bauman GS, et al. Case - 18F-DCFPyL-positron emission tomography/computed tomography (PET/CT) time of imaging. Can Urol Assoc J 2020. doi: 10.5489/cuaj.6984. Epub ahead of print.

9. Crook JM, Malone S, Perry G, et al. Twenty-four-month postradiation prostate biopsies are strongly predictive of 7-year disease-free survival: results from a Canadian randomized trial. Cancer 2009;115(3):673-9.

10. Pickett B, Kurhanewicz J, Coakley F, et al. Use of MRI and spectroscopy in evaluation of external beam radiotherapy for prostate cancer. Int J Radiat Oncol Biol Phys 2004; 60:1047-55.

11. Liu W, Zukotynski K, Emmett L, et al. Utilization of Salvage and Systemic Therapies for Recurrent Prostate Cancer as a Result of 18F-DCFPyL PET/CT Restaging. Adv Radiat Oncol 2020; 6:100553.

12. Young S, Liu W, Zukotynski K, et al. Prostate-specific membrane antigen targeted PET/CT for recurrent prostate cancer: a clinician's guide. Expert Rev Anticancer Ther 2021:1-15. 
Figures and Tables

Fig. 1. Selected axial prostate-specific membrane antigen positron emission tomography/computed tomography images of the pelvis show asymmetric focal uptake in the right posterior prostate gland.
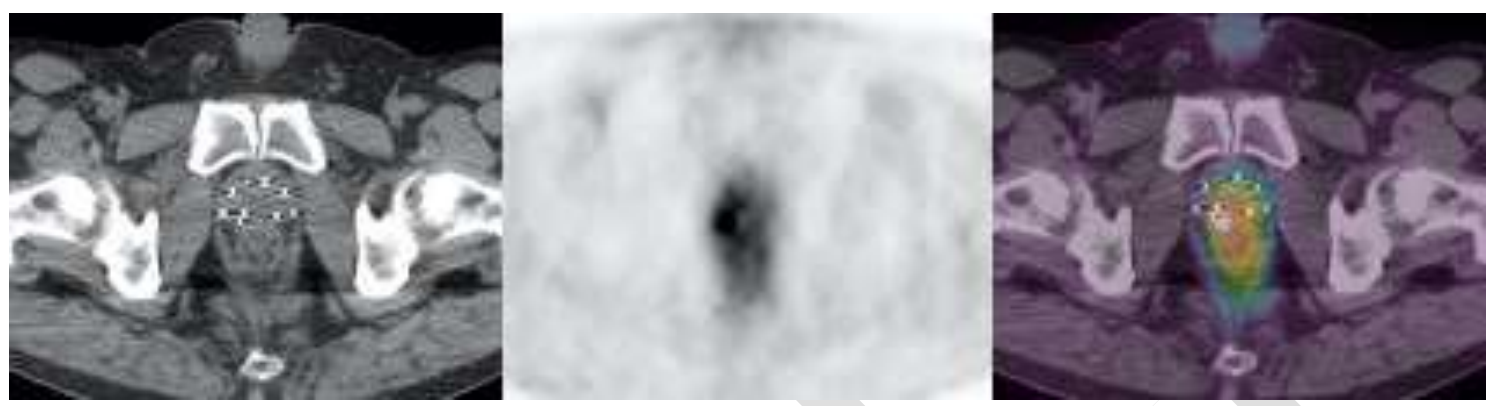

Fig. 2. Patient's prostate-specific antigen (PSA) changes during the time. Time 0 is when the patient was diagnosed with prostate cancer.

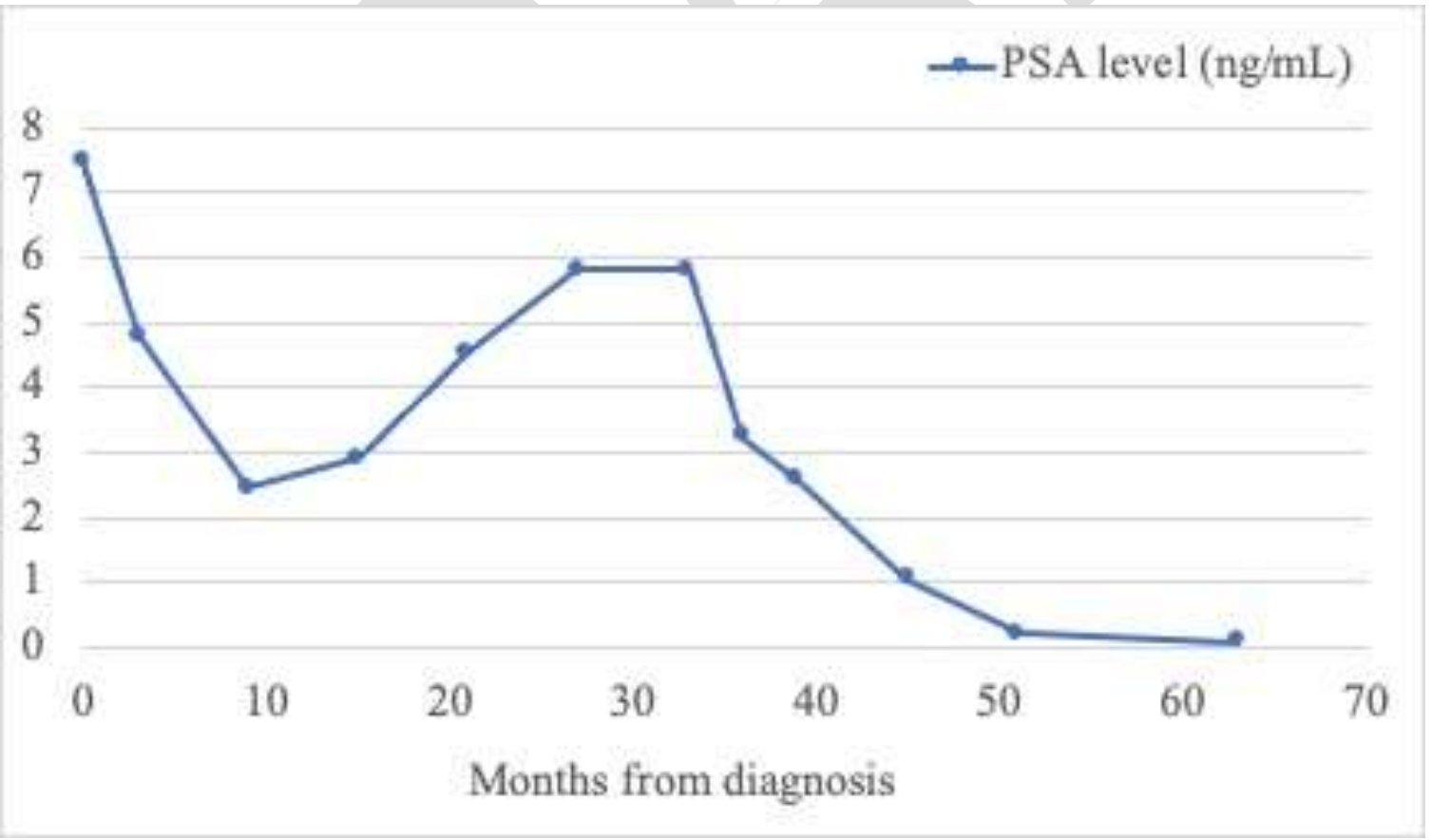

